\title{
X3D Field Trips for Remote Learning
}

\author{
Nicholas F. Polys \\ Advanced Research Computing \\ Virginia Tech \\ USA \\ npolys@vt.edu
Kathleen Meaney
School of Visual Arts
Virginia Tech
USA \\ kmeaney@vt.edu
}

\author{
John Munsell \\ Forest Resources and Environmental Conservation \\ Virginia Tech \\ USA \\ jmunsell@vt.edu \\ Benjamin Addlestone \\ Forest Resources and Environmental Conservation \\ Virginia Tech \\ USA \\ benj16@vt.edu
}

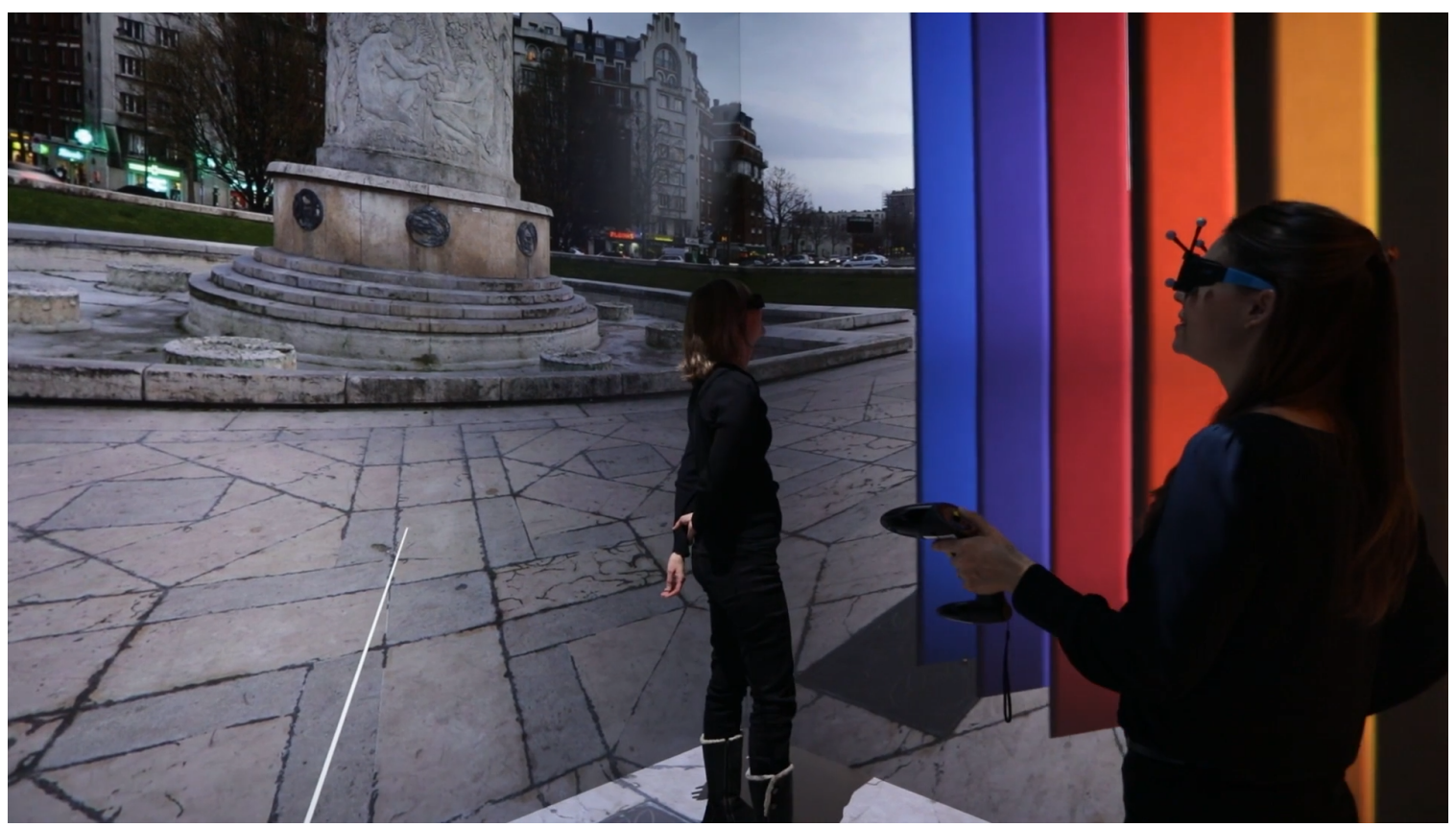

Figure 1: Bayer's Chromatic Gate sculpture set in a European square; immersively rendered in a 27.6 million pixel CAVE

\begin{abstract}
Combinations of immersive media and graphic portrayals can enable the human subjective sense of Presence. This paper collects our experiences and evaluations from six projects that use Extensible 3D (X3D) interactive graphics to deliver spatial experiences across the WWW. X3D enables the combination of spherical panoramas with 3D models and maps to visually transport users to a specific real location at a specific time. Remote users have access to these
\end{abstract}

This work is licensed under a Creative Commons Attribution International 4.0 License.

Web3D '21, November 8-12, 2021, Pisa, Italy

(c) 2021 Copyright held by the owner/author(s). Publication rights licensed to ACM ACM ISBN 978-1-4503-9095-8/21/11.

https://doi.org/10.1145/3485444.3487647 worlds through a Web-browser or other immersive device; local users in a CAVE can collaborate with natural physical gestures; . We reflect on the graphical and interactive requirements of these projects and provide guidance for future applications. In the face of physical lock-downs and distancing due to the CoVID pandemic, such platforms illustrate the opportunities and challenges in the design and delivery of spatial visualizations, especially for remote learning.

\section{CCS CONCEPTS}

- Computing methodologies $\rightarrow$ Rendering; Interaction.

\section{KEYWORDS}

Online courseware, 360-degree photography, Spherical Panoramas, Extensible 3D 


\section{ACM Reference Format:}

Nicholas F. Polys, John Munsell, Kathleen Meaney, and Benjamin Addlestone. 2021. X3D Field Trips for Remote Learning. In The 26th International Conference on 3D Web Technology (Web3D '21), November 8-12, 2021, Pisa, Italy. ACM, New York, NY, USA, 7 pages. https://doi.org/10.1145/3485444. 3487647

\section{INTRODUCTION}

When we visualize a place or a space, our intention is to 'transport' that user's consciousness to that location. Using 360-degree photo or video -spheres, one can photo-realistically capture and portray a specific location. These captures are discrete in that they come from a certain place at a certain time. In this sense, they meet the criteria of MirrorWorlds where there is ontological and temporal correspondence between the real world and the virtual world [Tilden et al. 2011]. Spherical panoramas can be quite compelling. Indeed their communicative power in combination of Head Mounted Displays (HMDs) has created what has been called 'The Empathy Machine' [Hassan 2020], [Bujić et al. 2020] when applied to real human situations such as depicting the vast squalor refugee camps in Jordan, or the destruction wrought by warfare.

Photosphere technology has been available for several decades, beginning with Apple's QuicktimeVR product [Chen 1995] and evolving recently with a wide variety of cameras and software. Photosphere-based Virtual Reality has been used for a number of local and remote educational applications (i.e. teaching subjects as varied as audio engineering [Cochrane 2005] or anatomy [Nieder et al. 2000] ).

Using 360-degree imagery for virtual field trips has been explored for Environmental Chemistry [Fung et al. 2019], Earth Sciences [Moysey and Lazar 2019], scientific methods [Harron et al. 2019], and historical sites [Burden et al. 2017] among others. Each of these papers emphasize the need for user agency (i.e. self-directed exploration). In addition, several noted that extended time (15 minutes) in an HMD was problematic for many users.

360-degree cameras have evolved drastically in terms of capture resolution and frame-rate. For most desktop and HMD display platforms, the captured image resolution is more than sufficient. However large, high-resolution projection displays, can exceed the capture resolution, making photospheres look grainy and out of focus. While camera manufacturers provide editors and Webbased software, they are constrained in their functionality and flexibility; specifically in their supported scene graph. Our projects use Extensible 3D (X3D), which is an ISO standard scene graph model with several encodings and programming APIs specified. For each location, an X3D scene was created; the X3D scenes were run in our CAVE and published on the WWW. For WWW distribution across browsers, X3DOM was employed [Behr et al. 2009], [Behr et al. 2011].

\section{PLACES AND SPACES}

Virtual Reality content and Web3D environments have seen a wide application across varied disciplines; complex spatial systems such as ecosystems can be effectively taught through interactive $3 \mathrm{D}$ worlds (i.e. [Metcalf et al. 2011]). Not only do spherical panoramic photography and video provide compelling context for 3D models,
3D spatial relationships becomes explicit in this media where the viewpoint and experience is under user control.

X3D provides the ideal language to build online educational applications where data comes from a variety of sources and is meant to be delivered to multiple computers and displays. A rich scene graph enables rich mashups including photospheres and scanned 3D models. Virtual field trip designers must match the media and perceptual information to the task and also consider the dual requirements of 3D spatial navigation and 2D page navigation. Over the years, we have collaborated on a number of projects that have tested the feasibility and effectiveness of Web3D for spatial visualization in education. Table 1 provides an overview of these projects and their functionality.

\section{Table 1: Projects and Scope}

\begin{tabular}{lcccc} 
Project & Photosphere & 3D Object & WWW & CAVE \\
\hline Site Capture & Y & Y & Y & Y \\
Site Visits & Y & Y & Y & Y \\
Class Design Project & Y & N & Y & Y \\
NASP certification & Y & N & Y & N \\
Chromatic Gate & Y & Y & Y & Y
\end{tabular}

\subsection{Site Capture}

Our first foray into this particular combination of media production was a scientific road trip to document the planting and management strategies for northeastern hardwoods and forest-farmed products. Polys and Munsell took a Ricoh Theta 360 camera and a structure.io sensor into the woods on an overcast day. At several selected locations, they captured photospheres and local 3D objects (Figure 2).

Photosphere captures of a site provide a host of important information. Not only are the trees of the local area put into perspective, but also the ground detail (forest floor) and canopy cover (overhead) are apparent. These are key factors in the assessment of plots for reasons of crowding and planting strategy. The 360 camera was mounted on a monopod at human eye-height (estimated average 1.6 meters). The operator hid behind trees and clicked frames through a bluetooth link on their mobile phone. The Ricoh Theta 360 has a still image resolution of $5376 \times 2688$ pixels.

2.1.1 Local 3D Objects. Particular objects in the nut grove / forest were of interest to capture and communicate to future audiences. Each photosphere image provides context and location for a specific management practice. For example, the use of shaded areas at various elevations to grow a variety of medicines and foods. Within the nut grove, mushrooms (Shiitake), Goldenseal, and Ramps were being cultivated. Using a structure.io sensor mounted in an iPad, the researchers captured several textured 3D objects, including mushroom logs, ramps, people, and interpretive signs (i.e. Figure 3). The structure.io software generated textured 3D models in several formats, including VRML. The models were scaled and included in the local photosphere. 


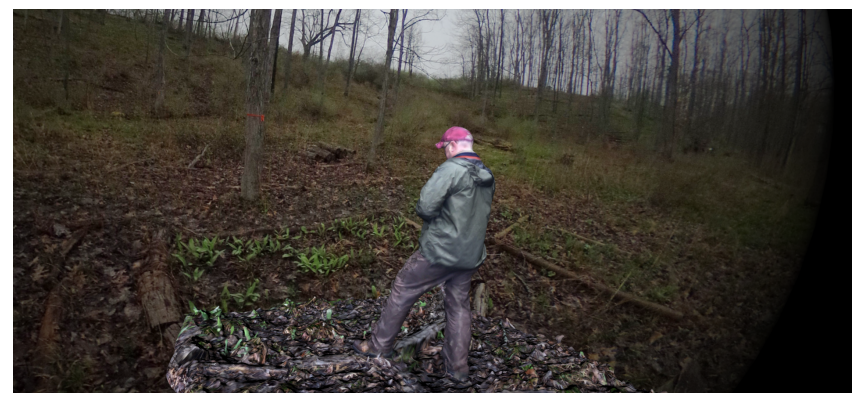

Figure 2: Nut Grove scan on location: captured 3D model of a researcher using the PlantShoe app at a Ramps patch, with photosphere as context ([Polys et al. 2020])

\subsection{Site Visits}

The 2018 Agroforestry conference was held in Blacksburg, Virginia. Our group prepared several virtual site visits for attendees through the Visionarium Lab. The virtual experience provided students a photo-realistic visit to several Agroforestry sites and provided the instructor a real-time, interactive environment for teaching. In this way, attendees not only visited a variety of farms (some hours away), but also were primed for the in-person on-site activities (Figure 4). The X3D scenes were also presented at the conference exhibit hall (Figure 5).

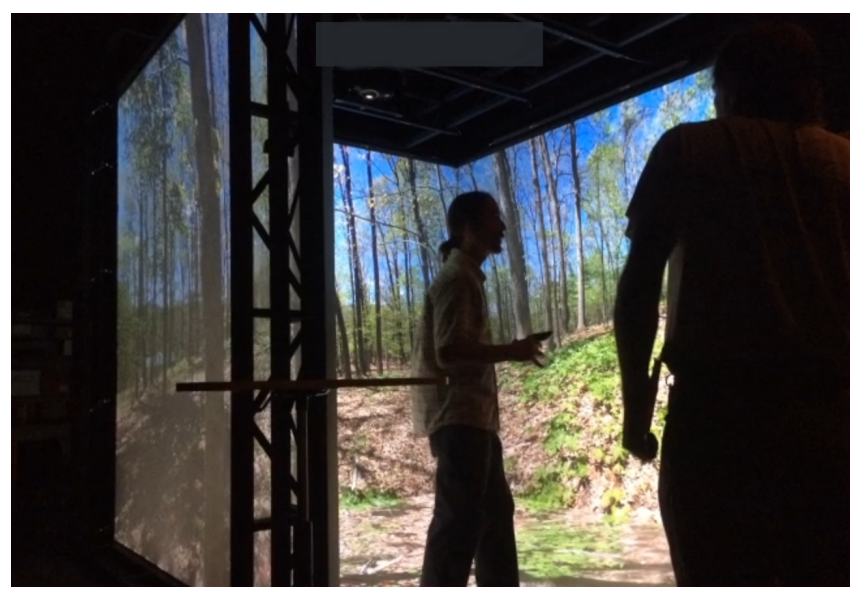

Figure 3: 2018 Agroforestry Conference Tutorial: virtual field trips delivered in the HyperCube by Eric Burkhart (Penn State) in the VT Visionarium Lab

\subsection{Class Design Project}

The College of Natural Resources offers a senior-level class, 'Agroforestry', which challenges students to understand and enable the forest ecosystem with secondary (non-timber) forest products. Such farming practices below the forest canopy include food to medicine with local native species such as GoldenSeal, Cohosh, Bloodroot, Ramps, and mushrooms.

As a capstone project in this class, students were challenged with a site design for the Old-growth forest stand on VT campus

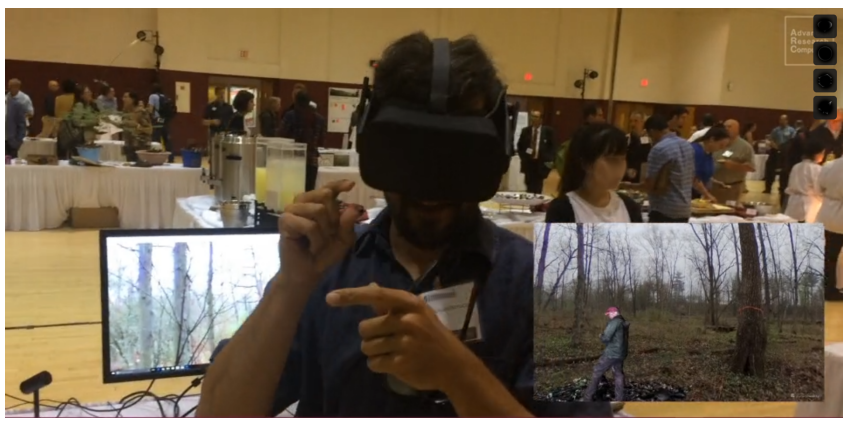

Figure 4: User travels to a site from the 2018 Agroforestry Conference with an HMD on the conference show floor

- 'Stadium Woods'. The property is notable in that it is: home to several native species and highly trafficked by football fans. The concept emerged for 'The Edible Edge', an Agroforestry design treatment for the thoroughfare peripheries that is productive for native species.

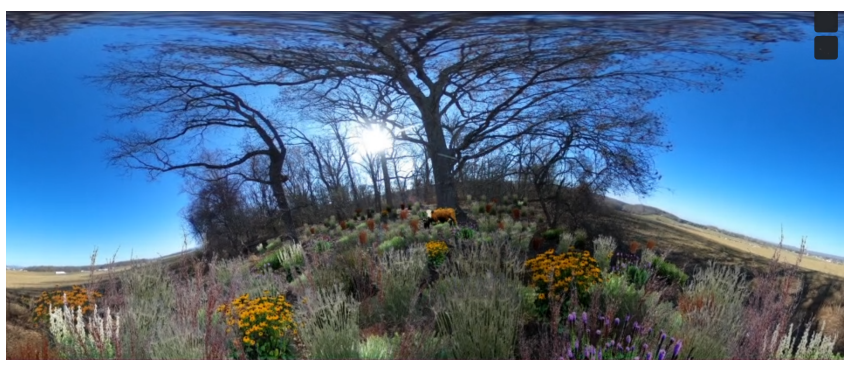

Figure 5: Student design solution in 2D image space: Equirectangular projection

After a tour and introduction to the property, students were given the Equirectangular image and trained with the US Forest Service's image editing software CanVis. Students developed several concepts and designs for the property on the $2 \mathrm{D}$ photosphere images (Equirectandular distortion). They reviewed and presented their designs as part of their final project (Figures 6 and 7).

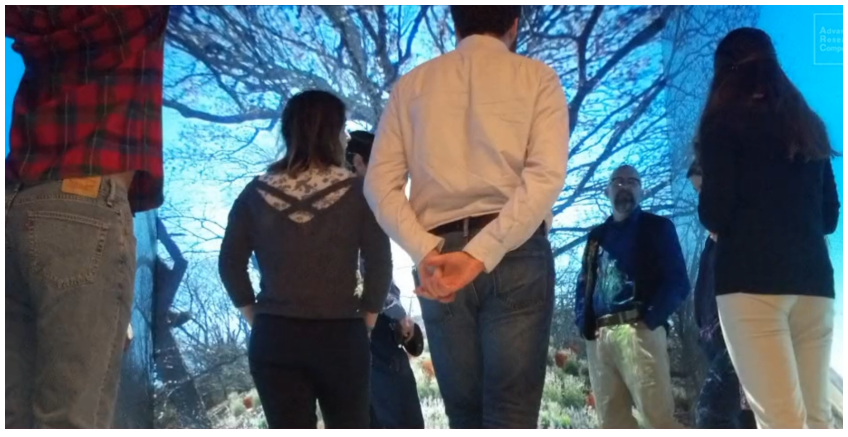

Figure 6: Class Design Review session in the CAVE 


\subsection{NASP Certification}

NASP stands for 'National Silviculture Program'. The US Federal Government provides funding for professional training of annual cohorts of up to 35 foresters across a broad range of silviculture topics and applications. Professionals are typically funded for an intensive two week session on Site Assessment and Best Management Practices (BMPs) for Silviculture (i.e. [Cristan et al. 2016] ). Managing forests for long and short -term productivity takes training, and each site is unique. As the 2019 planning evolved, Virginia Tech would host. Six forests were selected as case studies for the students to apply their new knowledge and translate it into a site-specific recommendation. For this project photospheres were captured with a GoPro MAX 360 at $16.6 \mathrm{MP}$.

CoVID changed this equation; sponsors and instructors had to scramble to implement a meaningful solution and deliver a course for the annual cohort. While there is no substitute for a real field trip, our goal was to make the best virtual field trip, accessible from anywhere at any time. According to the rubric of the course, (professional) students had to provide: evidence of critical site assessment and evidence-based recommendations. The course was administered through the Canvas learning management system with HTML pages for each site and plot.

Each site contains several plots, which are depicted in a clickable map in a minimizable tray at screen right. Each forest plot has a host of data associated with it and users can open another tray at screen left that contains the data table for all trees in that plot. Each tree has a unique number in both the photosphere and the data table. In addition, each plot has a link to a drone videosphere capture as well: the drone flew from plot center up through the canopy. Tree numbers were overlaid onto the video so that users could cross reference the trees with the data table (Figures 8 and 9).

At two sites, we also experimented with an additional capture and presentation technique for forest plots. Our goal was to help the students create an understanding of the spatial relationships among trees at each specific locality in the forest. Again, the plot center photosphere had numbered trees; however in the cases we created 5 spheres per plot: we also captured 360-degree images from the plot perimeter at the four cardinal directions. This combination of media and information allowed the students to virtually assess each site and provide management recommendations as part of their class assignments. They were also able to visit more types of forest than they could have physically: not only Appalachian hardwoods and mixed Piedmont forests, but also northern Pine stands (Maine).

\subsection{Chromatic Gate}

The School of Visual Arts has a course on Environmental and Exhibit Design. Herbert Beyer's Chromatic Gate sculpture is installed in a seaside park in San Diego. For a unit on the Bauhaus movement, we created an activity that transported the sculpture to different places and contexts. Specifically, we put Bayer's Chromatic Gate sculpture inside photospheres including: The Bauhaus School in Germany, the beach, a pier, by a river, in a European square, and on the planet Mars. In this case, the different Creative Commons photospheres available online provided lessons and discussion about the dialogue between a sculpture and its setting. A 3D model of

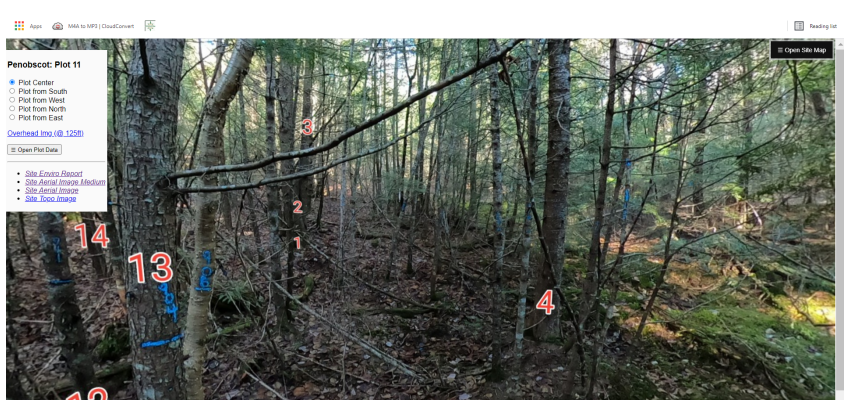

Figure 7: Penobscot forest plot 11

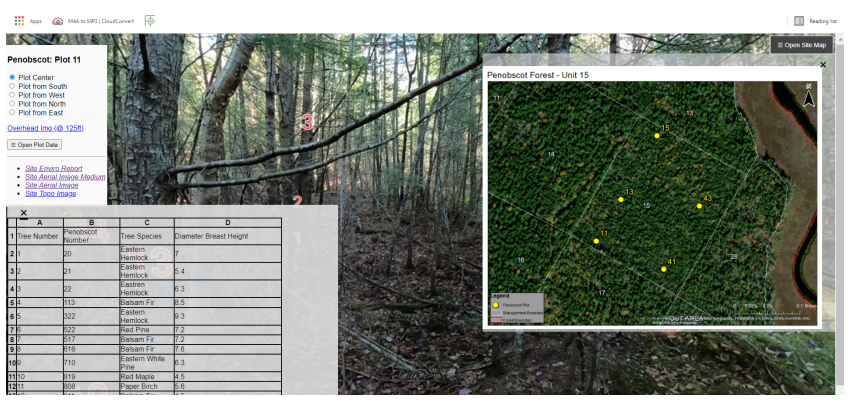

Figure 8: Penobscot forest plot 11 with detail and overview panels maximized

the Chromatic Gate sculpture was developed in centimeters in OBJ format and scaled to meters for X3D (Figure 10).

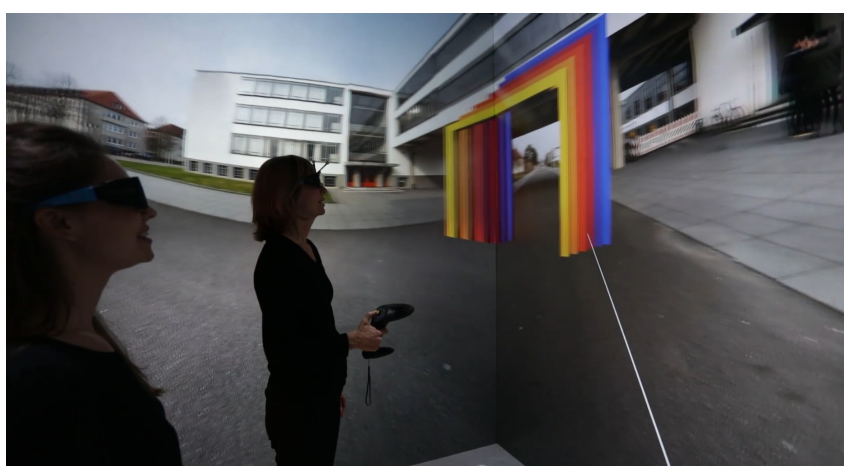

Figure 9: Bayer sculpture re-located to the Bauhaus School: immersive CAVE. Bauhaus school photosphere $\odot$ Dirk Wandel

\section{IMPLEMENTATION}

The visualization of place and space has become photorealistic through the use of commodity 360-degree spherical cameras. While camera vendors provide software for viewing the pictures, images are typically saved as jpgs in an equirectangular projection. Using a flexible scene graph language like X3D, authors can mash up and edit their worlds in the WWW programming idiom. Humanreadable files can be edited to include additional 3D models, to 
change the photo sphere image (by url), and to specify various aspects of lighting and navigation.

In this section, we describe the technical considerations in producing these remote field trips for a variety of applications and platforms.

\subsection{Graphics}

The first challenge is to map the photosphere image onto a spherical 3D geometry. While there is a native primitive node for Sphere in VRML and X3D, browsers are free to tessellate the sphere to any resolution. In order to achieve consistent rendering across X3D engines, we created a sphere with a radius of 18 meters and with 64 divisions in latitude and longitude. The primitive was then cast to a mesh. The mesh faces are defined by default with a counter clockwise rule, making the outside of the sphere the 'outside'. We reversed the triangle order (ccw ='false') so that only the inside of the sphere is drawn (solid = 'false'). This technique has the distinct advantage that users can see the photosphere image on the inside of the sphere even if they have navigated outside of the sphere bounds.

The default texture mapping for the Sphere node works well with Equirectagular projected images: the image texture is wrapped around the sphere, pinching the image at the top and bottom. For a few captures of natural landscapes, we did not notice that the geometric reversal (noted above) had to be matched with a texture transformation. When we saw text in the picture, we realized that the texture also has to be flipped where $<$ TextureTransform scale $=^{\prime}-11^{\prime}$ center $=^{\prime} .5 .5 \%$ ' .

The X3D scene graph enables authors to specify a variety of light types, including a headlight, directional light, point light, and spotlight. Most projects used the default headlight = 'true' and this was sufficient. For the Chromatic Gate project, a soft PointLight was also included and located by estimation to the sun's position in the various photospheres. As a scene graph, X3D can be rendered with a variety of technologies. Our CAVE (The VT HyperCube) runs the InstantReality engine across 8 projectors (16 displays for stereo), which are tiled and blended for a high-resolution immersive experience. For many of the forestry field trips described above, the content was simply the 360 degree imagery where students showed before and after their design treatment. In these cases, we observed that stereo rendering was not necessary since the sphere geometry is equidistant from the user's starting position. For the case of the Chromatic Gate and the Nut Grove site captures however, the 3D objects inside the photosphere did have 3D geometry and appearance and rendering the scene in (active) stereo did have a positive effect on the immersion and overall experience.

\subsection{Interaction}

Many photosphere viewing softwares render imagery in a similar way. They provide a virtual viewpoint at the camera's location and enable users to rotate their view of the sphere by heading and pitch with a mouse drag. We found that these methods are insufficient for a variety of applications, such as virtual field trips. The X3D scene graph enables authors to use several well-defined navigation modes, including EXAMINE, WALK, FLY, and LOOKAT. The default metaphor is EXAMINE where a center pivot point is defined and input device events are interpreted as translating or rotating relative to that point. While useful for viewing objects such as mushroom logs or sculptures from the outside-in at any angle, this navigation mode allows the camera to rotate in roll as well. As a result users can get disoriented and turned around easily. In addition, if a new pivot point is set by the user by accident, unintuative navigations can result, leaving the user lost in the world.

In scenes with multiple photospheres, we have experimented with several view animation techniques. In the basic version, we define a Viewpoint for each sphere as well as an overview Viewpoint. Users can use the scene events browser.nextViewpoint (PageUp key) and browser.previousViewpoint (Page-Down key) to step through the viewpoint list; depending on the nature of the field trip and lesson the spheres may be related to each other by location or other context (like McDaniel's Nut Grove - Figures 2, 3, and 5). In this case, we animate users out to the overview viewpoint before binding the next. For the Agroforestrty class, we provided a template scene where users would click large 3D arrows to toggle between the base spherical panorama and those of their design alternatives.

Our experience with X3D scenes in our CAVE led us to develop a solution that enables users to 'walk' around inside the photosphere. The default camera height in WALK mode (if not specified by the author) in X3D is 1.6 meters. The NavigationInfo node also provides parameters for the avatar's step height and collision distance. Therefore, we introduced a large invisible plane to serve as a floor or ground plane inside the photosphere. The photosphere's origin was set at $y=1.6$ (the initial viewpoint at head and capture height)) and the floor was set at $y=0$. We also set collision = 'false' on the photosphere Shape so that users could navigate through the sphere wall even in WALK mode. This setup enabled us to use our head tracking as well as wand-based navigation to explore the scenes in small co-located groups (Figures 1 and 10).

For the native WWW browser worlds published online, the X3DOM engine supports an experimental navigation mode called TURNTABLE. This navigation mode means to act like a 'Lazy Susan' rotating table for the scene. Users can rotate around a center point with heading and pitch rotation (no roll). In our first prototypes for the NASP Certification training program, we noticed that the TURNTABLE mapping interprets the mouse drag event as a change in the direction of the camera (relative to the pivot point). In this way, the user interaction feels to be manipulating the virtual viewpoint. In our design sessions with users, it was noted that the metaphor is hard to grasp for those not familiar with the 6 degree-of-freedom camera of 3D graphics. Instead, it was suggested that reversing the direction would be more natural because now the direction is interpreted as dragging the sphere around the fixed viewer. We provided both versions online to our stakeholders and held and informal poll. The results showed that the majority of users preferred the reverse TURNTABLE, which was then deployed in the class field trips.

\section{RESULTS}

\subsection{Agroforestry class}

Students were able to develop and publish their designs with the tools available. For example, the CANVis software provides a large 
image library of species and objects that can be added to an image. In addition, the layer and affine transformation tools make it easy to scale or skew the images into the correct perspective on the Equirectangular projected images. Thus, students worked in 2D and updated the image urls in their X3D files. The final class Design presentations were given by students in the VT HyperCube (Figure 7). Video.

\subsection{NASP Certification}

We encountered several challenges in the deployment of this certification program though a remote online class. Most important to note is the fact that we did not have their undivided attention as we would have had if the class was held in the same room or field site together. Specifically, they were still in their usual modes of operation (with co-measurate obligations to office, children, timezone, etc). Students were challenged to survey several forest sites with diverse conditions and make recommendations based on the principles they learned in the synchronous class.

We checked in with the students at the beginning of the course and asked, "How have you adjusted your approach to learning in NASP due to COVID-19 restrictions on in-person learning?" before during, and then after the course. Prior to the course, we saw a range of attitudes amongst the participants: some were skeptical of how effective the virtual approach would be for such field-based coursework, some were familiar with computer fatigue, others were open-minded as shown in the following comment "I am not sure I have adjusted my approach as my entire NASP experience has been virtual. I have been attempting to keep an open mind to this type of learning experience and get as much as I can out of it."

In the middle of the course, after a few exercises with the X3D photospheres, we asked students about their experiences with the course and site survey media. Of the 22 responses, 19 had positive reactions to the photosphere site assessment ( 86 percent). There were some notable qualifications. These comments demonstrate a generally positive reception to the X3D field trips. Of those 19 positive comments, two specifically mentioned how the pentasphere site method helped them get better perspective on the plot (rather than one sphere per plot); three mentioned how it took time to get used to experiencing a site this way. Here are some representative positive comments: "I enjoyed the AR/VR imagery as it really helped give us a sense of being in the woods" and "excellent way to see a site when you can't get there".

Three students strongly noted other difficulties and limitations. Two of these for example, felt that the resolution of the imagery and video was too low to gain a confident feel for the locations. One student noted the difficulty of navigating across plots while assessing treetops. These comments help us identify weaknesses that we could improve for the next version; specifically, the learnability of the interface, consistent display across screen sizes (resolution and scale), and better virtual navigational techniques that can support task-based strategies.

After the course, we asked again student perspectives on the course and use of photospheres. Of the 7 comments we received, we can see that the students were generally satisfied with the virtual field trip component, even though it was not considered equivalent to actual visits in the field. Two quotes capture this sentiment nicely:
"The diverse methods of content delivery and presentation was great for a remote class. The group work, ARVR, instructor organized and informal ( $Q$ and $A)$ time and availability, and individual work all formed a comprehensive learning program." and "I really enjoyed the $V R$ aspect of the course. I didn't think it was possible to understand and comprehend the material in a virtual setting.".

\subsection{Chromatic Gate}

Here are some student comments from the class; the Video is Here.:

(1) "What really jumped out at me was how scale feels digitally $v$ in real life. I automatically up scaled the size of items that I saw digitally. I didn't really consider these pieces being at a small scale. This is probably because of how it was presented online. Everything was scaled to match a screen so I didn't really think of how these look next to one another or what the actual dimensions might be. It would be pretty odd for everything to be similarly sized like the computer presented."

(2) "It is always interesting to me seeing the actual size of a piece compared to a $2 \mathrm{D}$ image from a screen. Scale is everything, well maybe not everything but it's certainly important, and the computer takes that factor out of the question. Also, it was interesting to see how the pieces were organized throughout the space, rather than simply uploaded into an online collection"

(3) "Another thing I learned during this process was how different things can seem online vs in real life. Specifically speaking about the Herbert Bayer exhibit, I found that scale was often misinterpreted when all of the artifacts were displayed at the same size on my screen. Despite having the dimensions in the metadata, I automatically assumed that they were all about the same size. This assumption was proved wrong when I saw pictures of the actual exhibit. This is something I tried to keep in mind for the other collections/research for the rest of this project."

(4) "History was one of my worst subjects because normally I don't have much interest in it, but learning about history in this way was a lot more interesting."

\section{CONCLUSIONS AND FUTURE WORK}

We have described a multi-year effort to design and develop photorealistic Web3D experiences for the visualization of place and space. Our approach has evolved through several applications, audiences, and their requirements. With the recent CoVID pandemic, such technologies for remote fields trips are even more valuable. While VR will never be a perfect substitute for real experience in the field, we can match our rendering cues and interactions to specific user tasks with good subjective and pedagogical outcomes. In addition, having virtually visited a place enables the user to 'prime' themselves so that they can spend their time more efficiently once onsite in real life.

We have shown several X3D techniques to create photo-realistic virtual field trips and reported their qualitative effectiveness. The WWW and X3D provide designers a rich palette of user interface possibilities. Indeed, User Interfaces can be customized to include rich multimedia content and look and feel. The flip side to this flexibility is that users may have to re-learn metaphors and interactions of the application. This also means that currently, authoring 
these field trips requires editing the X3D scene in order to tailor the environment for the activity.

Multi-disciplinary teams can provide a great advantage in the design of such environments. We used User-Centered design activities and Cognitive Task Analysis to develop our requirements, design, and evaluation criteria. Paying attention to the instructors' recommendations and their knowledge of their students was crucial. Thus we attribute our success to a close dialogue among collaborators and the willingness to try something new. For example, in the cases of photosphere-only scenes, stereo rendering was more a harm than a help. In addition, the stakeholders' background and their available metaphors drove us to modify the navigation for usability in immersive CAVEs and for Desktops and WebVR platforms. With the technology platforms ready and able, the challenge now comes to $3 \mathrm{D}$ authors and designers to use them creatively and advantageously. Open Web3D standards such as X3D provide a rich palette for instructional design and educational media.

Future research should examine user judgements of size and depth in spherical panorama environments. Calibrating capture and viewing platforms can support improved confidence in the assessment and design for scale in real spatial context. The NASP prototype had three levels of scale portrayed(site, plot, tree). Students indicated that spatial knowledge of a plot was still difficult to integrate from the different (separate) views. Similar to our prior work on IRVEs, user feedback indicates that we should favor Association over Occlusion; in this application, practically speaking, this means future NASP versions should work to overlay DBH and species detail information over the photosphere view to provide focus+context of tree information inside the photosphere view rather than in separate overview-and-detail panels. Finally, as more Web3D platforms support WebXR, these virtual fieldtrips can be used in augmented and mixed reality contexts with commodity hardware.

\section{ACKNOWLEDGMENTS}

We would like to thank Ellie Nikoo, Dayoung Kim, and Catalina Lemus for their work processing photospheres, also Carolyn Commer as immersive explorer, Phat Nguyen as 3D Bayer modeller, and David Franusich as videographer. This work was supported in part by the Smithsonian Learning Lab, the US Forest Service, and Virginia Tech's Institute for Creativity, Arts, and Technology and its Advanced Research Computing group.

\section{REFERENCES}

Johannes Behr, Peter Eschler, Yvonne Jung, and Michael Zöllner. 2009. X3DOM: a DOM-based HTML5/X3D integration model. In Proceedings of the 14th international conference on $3 D$ web technology. 127-135.

Johannes Behr, Yvonne Jung, Timm Drevensek, and Andreas Aderhold. 2011. Dynamic and interactive aspects of X3DOM. In Proceedings of the 16th International Conference on 3D Web Technology. 81-87.

Mila Bujić, Mikko Salminen, Joseph Macey, and Juho Hamari. 2020. "Empathy machine" how virtual reality affects human rights attitudes. Internet Research (2020).

DJH Burden, T Argles, S Minocha, J Rock, S Tilling, and P Wheeler. 2017. Fieldscapescreating and evaluating a 3D virtual field trip system. (2017).

Shenchang Eric Chen. 1995. Quicktime VR: An image-based approach to virtual environment navigation. In Proceedings of the 22nd annual conference on Computer graphics and interactive techniques. 29-38.

Thomas Cochrane. 2005. Interactive QuickTime: Developing and evaluating multimedia learning objects to enhance both face-to-face and distance e-learning environments. Interdisciplinary Journal of E-Learning and Learning Objects 1, 1 (2005), 33-54.
Richard Cristan, W Michael Aust, M Chad Bolding, Scott M Barrett, John F Munsell, and Erik Schilling. 2016. Effectiveness of forestry best management practices in the United States: Literature review. Forest Ecology and Management 360 (2016), 133-151.

Fun Man Fung, Wen Yi Choo, Alvita Ardisara, Christoph Dominik Zimmermann, Simon Watts, Thierry Koscielniak, Etienne Blanc, Xavier Coumoul, and Rainer Dumke. 2019. Applying a virtual reality platform in environmental chemistry education to conduct a field trip to an overseas site.

Jason R Harron, Anthony J Petrosino, and Sarah Jenevein. 2019. Using virtual reality to augment museum-based field trips in a preservice elementary science methods course. Contemporary Issues in Technology and Teacher Education 19, 4 (2019), 687-707.

Robert Hassan. 2020. Digitality, virtual reality and the 'empathy machine'. Digital fournalism 8, 2 (2020), 195-212.

Shari Metcalf, Amy Kamarainen, M Shane Tutwiler, Tina Grotzer, and Chris Dede. 2011. Ecosystem science learning via multi-user virtual environments. International fournal of Gaming and Computer-Mediated Simulations (IFGCMS) 3, 1 (2011), 86-90.

Stephen MJ Moysey and Kelly B Lazar. 2019. Using virtual reality as a tool for fieldbased learning in the earth sciences. In Interdisciplinary Perspectives on Virtual Place-Based Learning. Springer, 99-126.

Gary L Nieder, Jane N Scott, and Mark D Anderson. 2000. Using QuickTime virtual reality objects in computer-assisted instruction of gross anatomy: Yorick-the VR Skull. Clinical Anatomy: The Official fournal of the American Association of Clinical Anatomists and the British Association of Clinical Anatomists 13, 4 (2000), 287-293.

Nicholas Polys, Peter Sforza, and John Munsell. 2020. PlantShoe: Botanical Detectives. In HCI Outdoors: Theory, Design, Methods and Applications. Springer, 119-136.

Dan Tilden, Ankit Singh, Nicholas F Polys, and Peter Sforza. 2011. Multimedia mashups for mirror worlds. In Proceedings of the 16th International Conference on $3 D$ Web Technology. 155-164. 